\title{
Nodal Surfaces in Molecular Wave Functions
}

\author{
KARL F. HERZFELD \\ Department of Physics, Catholic University of America, Washington, D. C.
}

\begin{abstract}
One electron molecular orbitals for mobile electrons, subject to the potential energy of a molecular skeleton, are considered. If the atoms are far apart, the orbitals can be built up exactly from $2 p$ atomic wave functions. The molecule in natural size is formed by adiabatic approach. It is shown that all the nodal surfaces reach into infinity, if the atoms are all alike, but that closed nodal surfaces may exist, if they are not. It is proved that the number of electrons inclosed in nodal surfaces is invariant against adiabatic change. Nodal surfaces for antisymmetrized molecular orbitals are then discussed.
\end{abstract}

\section{INTRODUCTION: DISCUSSION OF THE PROBLEM}

$I^{\mathrm{F}}$ $\mathrm{F}$ one solves the wave equation of a single valence electron for an atom, subjecting the electron to a field of central symmetry, the variables can be separated. In that case, the nodal surfaces can be divided into three mutually orthogonal families, and any solution is characterized by three quantum numbers; the energy increases if any one of the quantum numbers is increased.

The general problem, in which the wave equation is not separable, has first been touched by Sommerfeld. ${ }^{1}$ Wintner ${ }^{2}$ has then extended Sturm's oscillation theorem to this case. His investigation is applicable to the case of one electron wave functions and gives the following result. Consider, for a given wave function, a region, bounded by nodal surfaces and possibly infinity, in which the wave function does not change sign. Then a wave function with higher energy will change sign in that region at least once. Moffit and Coulson ${ }^{3}$ have investigated the nodal surfaces in hybridized (and therefore non-separable) atomic wave functions. Hund ${ }^{4}$ has considered diatomic molecules as being formed either by separating parts of a "united" atom, or by bringing an atom and an ion together. In this case there is no change in the number of nodal surfaces, but this procedure has not been applied to the consideration of the usual molecular orbitals in polyatomic molecules.

In this paper we are mainly interested in the nodal surfaces of wave functions of mobile electrons (conjugated double bonds) where the interaction is not as strong as in $\sigma-\sigma$ bonds. To permit a discussion of nodal surfaces in the usual sense, the wave function must be real, except for a space independent phase factor. In the present problem, this is always the case for nondegenerate wave functions. For degenerate functions, it it always possible to form real linear combinations.

If one uses one electron molecular orbitals built up from atomic wave functions, the problem of finding the

${ }^{1}$ A. Sommerfeld, Partielle Differentialgleichungen der Physik (Akademische Verlagsgesellschaft, Leipzig, 1947).

2 A. Wintner, J. Chem. Phys. 16, 405 (1948).

${ }^{3}$ W. E. Moffit and A. C. Coulson, Phil. Mag. (VII) 38, 634 (1947).

${ }^{4} \mathrm{~F}$. Hund, Handbuch der Physik (1933), second edition, Vol. $24, \mathrm{I}$. coefficients is analagous to that of finding the modes of vibration of a properly selected arrangement of coupled particles. The author has pointed out in a previous paper ${ }^{5}$ that in such a vibration problem one can also order the states into a series with ascending number of nodes, each number being represented, and that the frequency (for the molecules: the energy) increases with the number of nodes. This however says only something (in the case of the wave function) about the number of nodal points on the lines joining the atoms, not about the number of nodal surfaces. For example, in the wave function for a four carbon molecule $C_{1} C_{2} C_{3} C_{4}$ which has two nodal points, namely, the one in which the sign of the coefficients is +--+ , there might be a single closed surface inclosing the two central carbon atoms.

The present author has investigated ${ }^{6}$ the ways by which the number of nodal surfaces of a solution might change if the boundaries or the wave equation is changed adiabatically. This paper is an attempt to discuss the shape and number of the nodal surfaces.

\section{ONE ELECTRON ORBITALS}

\section{(a) Assumptions, Notations}

The following assumptions are made: We consider one electron in the field of the molecular skeleton; this field is considered as originating from charges in the close neighborhood of the atomic nuclei. (This is a good approximation for the field due to electrons in inner shells and involved in $\sigma-\sigma$ bonds, but not for that part of the field due to other mobile electrons.)

We select an arbitrary origin within the molecule, an arbitrary direction of the polar axis, and introduce atomic units (the radius $R$ measured in Bohr radii, the energy $E$ measured in the ionization energy of the ground state of $H$ ). Define $\beta_{j}=Z_{j} / n_{j}{ }^{* 2}$ where $Z$ is the effective charge and $n_{j}^{*}$ the effective quantum number of the $2 p$ atomic wave function of the $j^{\text {th }}$ atom. Furthermore we introduce a function

$$
u=\psi / R \text {. }
$$

${ }^{5}$ K. F. Herzfeld, Chem. Rev. 41, 233 (1947). See also: J. R. Platt, "Classification of spectra of catacondensed hydrocarbons," J. Chem. Phys. 17, 484 (1949). 6 K. F. Herzfeld, Zeits. f. Naturf. (Sommerfeld-heft) 3a, p. 457
(1948). 
Let

$$
\begin{gathered}
L=\frac{\partial}{\partial \mu}\left(1-\mu^{2}\right) \frac{\partial}{\partial \mu}+\frac{1}{1-\mu^{2}} \frac{\partial^{2}}{\partial \varphi^{2}} \\
(\mu=\cos \vartheta)
\end{gathered}
$$

be the Legendre operator. The Schroedinger equation then takes the form ${ }^{7}$

$$
\partial^{2} u / \partial R^{2}+\left(1 / R^{2}\right) L u+(E-V) u=0 .
$$

Here $E$ is a negative quantity.

\section{(b) Limiting Behavior at Large Distance}

Draw a sphere of radius $R^{\prime}$ around the origin which fulfills the following conditions:

$$
|E|>\left|V\left(R^{\prime}\right)\right| \text {. }
$$

At distances several times larger than the molecular dimensions, $V$ will represent the potential of a pole, and a few Bohr radia beyond the farthest atom will in general be sufficient to satisfy (2). Furthermore for the majority of directions, there is then also

$$
|E||u|>1 / R^{2}|L u| \text {. }
$$

(3) is not valid near the angular nodes.

If (2) and (3) are satisfied, it follows from (1) that $\partial^{2} u / \partial R^{2}$ has the same sign as $u$. If one goes out along a radius, in a direction for which (3) is satisfied, it follows then one cannot find ${ }^{8}$ for such a direction the value $u=0$ beyond $R^{\prime}$. In other words, beyond $R^{\prime}$ no nodal surface can cut a radius vector for any direction for which (3) is satisfied; the farther out one goes, the fewer the directions in which (3) is not satisfied.

One can, by using the $B-K-W$ method, write approximately for $R>R^{\prime}$

$$
u=C \exp \left(-(-E)^{\frac{1}{2}} R\right)\left\{B+Z^{\prime} / Z E \ln R\right\} S,
$$

where $S$ depends ${ }^{9}$ on the angles only and $Z^{\prime}$ is the total charge on the skeleton.

\section{(c) Atoms Far Apart}

Assume the atoms arranged in a pattern geometrically similar to the molecular pattern but with all nuclear

\footnotetext{
${ }^{7}$ In the following it is assumed that $\mathrm{V}$ is a potential energy. If it includes a Fock operator, $b$ and $c$ still hold but $d$ and III have not been proved.

${ }_{8}$ This implies that $u$ changes sign on crossing a node. That has been proved in reference 6 .

${ }^{9} S$ will in general not be a spherical harmonic. To force a separation into solutions with spherical harmonics of different order, one would have to go close enough so that the term $\left(1 / R^{2}\right) L(u)$ matters and then the asymptotic solution is not applicable any more. To illustrate this, consider an imaginary molecule of four atoms at the corners of a square of side $2 a$ in the $x-y$ plane, very large compared to the Bohr radius (see $c$ ), and write a one electron molecular orbital for the mode which has the $x z$ plane as nodal plane. The function is, for $R \gg a$,

$$
\psi=A z e^{-\beta R} \sinh (\beta a \sin \vartheta \cos \varphi) \cosh (\beta a \sin \vartheta \sin \varphi) .
$$

In other words, however far away one goes, the angle dependence is given by the same series of spherical harmonies. The dependence on $R$ agrees with (4) since for this large value of $R$ the energy is not different from that of a single atom. distances multiplied by the same factor $\gg 1$. Then all the nuclear distances will be many Bohr radii, and the one electron molecular orbital will be very exactly given by a linear combination of unperturbed atomic $2 p$ functions.

The only node of the atomic wave function is the symmetry plane of the (atomic) spherical harmonic. These will combine in the molecular orbital to a single open surface going to infinity. Let the molecular orbital have $s$ nodal points, as determined by the changes in sign of the coefficients, and follow a nodal surface (called here molecular node) outward. It is entirely possible that inside the extended molecule, a single molecular nodal surface passes through more than one nodal point. This will be mainly determined by the molecular symmetry (degeneracy), as illustrated in the case of benzene (see reference 5).

For the behavior outside of the molecule, two cases must now be distinguished.

$(\alpha)$ All the atoms involved are alike, i.e., their atomic wave functions decrease in the same manner with the distance from the atomic nucleus (same $\beta_{j}$ ). Since at a nodal surface the atomic wave functions from two or more atoms cancel and since the atomic wave functions are now practically unchanged and since finally the atomic distances in the magnified arrangement are many times the Bohr radius, it follows that the nodes will not approach any atomic nucleus closer than many Bohr radii, but will instead be nearly equidistant from the atomic nuclei involved. But according to II $b$, no nodal surface can be cut by a radius vector at large distance over a wide steric angle, so that all nodal surfaces which cross nodal points must go to infinity.

It is imaginable that closed nodal surfaces exist which do not cross a nodal point. Such surfaces could either lie entirely within an extended (non-linear) molecule or could be small and lie at great distance in a direction violating (3). However this possibility has never been encountered and seems very improbable.

It is possible to write the general wave function for distances $R>R^{\prime}$. Let $c_{j}$ be the coefficient of the $j^{\text {th }}$ atomic $2 p$ function in the molecular orbital, $\gamma_{j}$ the angle between $R$ and the axis of the $p$ function, and $x_{j}, y_{j}, z_{j}$ the coordinates of the $j^{\text {th }}$ nucleus. Then

$$
\begin{aligned}
\psi= & A R e^{-\beta R}\left\{\sum_{\gamma} c_{j} \cos \gamma_{j}\right. \\
& \left.\times \exp \left[-\beta z_{j} \cos \vartheta-\beta \sin \vartheta\left(x_{j} \cos \varphi+y_{j} \sin \varphi\right)\right]\right\} .
\end{aligned}
$$

$(\beta)$ This argument does not hold if different atoms are involved. If one writes the exponential factor in the atomic wave function $e^{-\beta_{j} R}$ one sees that nodal surfaces may be closed. Consider an imaginary linear molecule $A B A B$, and the one electron molecular orbital which has as signs of the coefficients +-+- . Assume $\beta_{B}>\beta_{A}$. In the immediate neighborhood of an atom, 
the sign of the wave function is determined by the wave functions of that atom, i.e., near an atom $A$ it has the sign of $+z$, near an atom $B$ the sign of $-z$. At large distances however the wave functions with smaller $\beta$ prevail and the sign is that of the $A$ 's, i.e., $+z$. Therefore the $B$ atoms are enclosed by closed nodal surfaces.

\section{(d) Natural Size of the Molecule}

If the atoms are brought closer together until the natural size of the molecule is reached, the best atomic wave functions from which to build a one electron molecular orbital will not be the unmodified $2 p$ function any more; nonetheless, one can follow the fate of the wave functions by the arguments developed in reference 6 . If only $2 p$ functions of one kind are involved (Sec. $c, \alpha$ ) one starts out exclusively with nodal surfaces going to infinity, asymptotically parallel to $R$ (excluding the possibility of closed nodal surfaces not passing through a nodal point). Because of (3), two such nodal surfaces may not, upon decrease of the atomic distance, join in infinity, and therefore two nodal surfaces may not merge upon further decrease of the molecular size (this differs from the case of a boundary problem with finite boundary). Nodal surfaces may approach each other and cross, but this process does not change their total number. Therefore, the number of nodal surfaces cannot decrease. The only imaginable way by which the number may increase would be if a nodal surface, at distances not very much greater than $R^{\prime}$ ( $R^{\prime}$ decreases with the size of the "molecule"'), formed a small sack, which would then be separated from the original surface through "Abschnuerung," forming an additional nodal surface, which would be small, outside the molecule and closed. This process however seems very improbable.

In the case in which not all atoms have the same $\beta$ and closed nodal surfaces exist, this argument is not applicable, since the total number of closed surfaces may increase by the formation of a "waist" which goes over in a double cone, which then leads to separation by "Abschnuerung," or two closed surfaces may merge, decreasing the total number of nodal surfaces.

Similar processes might conceivably occur when $3 p$ atomic functions are involved, since these have inner closed nodal surfaces.

To sum up: The nodal surfaces in a one electron molecular orbital all go to infinity provided the separate atoms had all $2 p$ wave functions of the same size. One of the nodal surfaces originates from the nodal plane of the individual atoms, the number of the other molecular nodes can be found by considering the different modes of coupled oscillators. ${ }^{10}$ Asymptotically all the nodal surfaces are parallel to $R$.

If other than $2 p$ functions are involved, or if not all

${ }^{10}$ I.e., if $N$ atoms are involved, there exist modes with any number of molecular nodal surfaces from zero to a maximum which is $N-1$ in absence of degeneracy, lower in the presence of degeneracy. atoms have functions of the same size, there will exist closed nodal surfaces, at least in some modes. In that case it has not been possible to make general statements about the number of nodal surfaces.

It follows from the preceding together with Wintner's arguments . . . for the case in which all $2 p$ functions are alike, no two states of different energy can have the same number of nodal surfaces, and that this number increases by one if one goes to the state of next higher energy. On the other hand, the four states of a molecule $A B B A$ have the following molecular nodes: None, a plane node bisecting the molecule, a closed node enclosing $B B$, a plane plus a closed node.

\section{A THEOREM ON THE CHARGE BOUNDED BY NODAL SURFACES}

The following theorem will be shown to hold, without limitation to molecular wave functions:

Consider a region bounded by nodal surfaces and possibly reaching to infinity. If the nodal surfaces are changed adiabatically, the amount of charge contained in the region is conserved, at least if the wave function is non-degenerate.

Call the amount of material (electrons) involved $q$.

$$
q=\int \psi^{*} \psi d \tau
$$

the integral being extended over the region defined above. Then

$$
d q / d t=\int \partial / \partial t\left(\psi^{*} \psi\right) d \tau+\int \psi^{*} \psi W_{n} d S
$$

where $S$ is the boundary surface and $W_{n}$ its velocity in the direction of the outer normal. Using the continuity equation and Gauss's Theorem, one finds

$$
\frac{d q}{d t}=\int\left\{\frac{h}{4 \pi m i}\left(\psi \operatorname{grad}_{n} \psi^{*}-\psi^{*} \operatorname{grad}_{n} \psi\right)+\psi^{*} \psi W_{n}\right\} d \tau
$$

According to the adiabatic law, ${ }^{11}$ a system described by a wave function in which a parameter is slowly varied is described at every instant by the same wave function with the momentary value of the parameter. Therefore if in the case of a non-degenerate state with real wave function (apart from a phase factor) a parameter is slowly varied, the wave function will stay real, and on the momentary nodal surface and in infinity $\psi$ will disappear, so that $d q / d t=0$.

Degenerate wave functions can, as mentioned in I, be combined to real ones; in this case coefficients may so change in the adiabatic process that one ends up with a linear combination of degenerate eigen functions which is complex. However in the process described in

\footnotetext{
${ }^{11}$ H. A. Kramers, Grundlagen der Quanten Theorie (Akademische Verlagsgesellschaft, Leipzig, 1933) p. 215.
} 
II, the symmetry of the configuration is maintained, and therefore the adiabatic process will not split the degenerate eigen functions, and a real combination will remain real.

For the case of the one electron molecular orbitals treated in II $c$, one considers a region bounded by nodal surfaces and possibly by infinity, in which $\psi$ does not change sign. For this region $q=\frac{1}{2} \sum c_{\gamma}{ }^{2}$.

Here $c_{j}$ is the normalized coefficient of the $j^{\text {th }}$ atomic $2 p$ wave function in the particular mode, and the summation extends over the $j$ 's corresponding to those nuclei which are situated on the boundary of the region. The formula remains exact for the natural size of the molecule, in spite of the fact that then the exact molecular orbital cannot be built up from $2 p$ atomic functions. The $c_{j}$ continue to have the "naive" values which are correct for the enlarged molecule.

In the example discussed in Section IIc, $\beta$ ) there is exactly $\frac{1}{8}$ of an electron in the region around an atom $B$, bounded by the $x y$ plane and the closed nodal surface.

\section{ANTISYMMETRIZED MOLECULAR ORBITALS}

Assume one builds now antisymmetrized molecular orbitals. Call $N$ the number of electrons involved and assume a resultant total spin $\sigma$; therefore assign to $\frac{1}{2}(N+\sigma)=K$ one electron molecular orbitals the spin $\alpha$, to $\frac{1}{2}(N-\sigma)$ the spin $\beta$; number the first from 1 to $K$, the second from $K+1$ to $N$. The antisymmetrized molecular orbital is then:

$$
\begin{aligned}
\Psi=\frac{1}{M} \sum P(-1)^{P} \psi_{1}(1) \alpha \psi_{2}(2) \alpha \cdots \\
\psi_{K}(K) \alpha \psi_{K+1}(K+1) \beta \cdots \psi_{N}(N) \beta,
\end{aligned}
$$

where $P$ as usual signifies all the electron permutations.

It is clear that one has to consider $\Psi$ in the $3 N$ dimensional configuration space $x_{1} y_{1} \cdots z_{N}$. A nodal surface is a manifold of $3 N-1$ dimensions. However one can easily see that a node for $\Psi$ has no meaning.

Instead we divide the $N$ ! permutations into subgroups. Such a subgroup, which shall be called a spin island, is defined as a permutation which exchanges all electrons with $\alpha$ spins among themselves and all electrons with $\beta$ spins among themselves, but not electrons with $\alpha$ spins with those of $\beta$ spins. Each spin island has $K !(N-K) !$ terms and there are $[N ! / K !(N-K) !]$ spin islands. In a particular spin island, one can write, apart from the normalization factor,

$$
\begin{aligned}
& \alpha^{K}\left\{\sum P^{\prime}(-1)^{P^{\prime}} \psi_{1}(1) \psi_{2}(2) \cdots \psi_{K}(K)\right\} \\
& \quad \times \beta^{N-K}\left\{\sum P^{\prime \prime}(-1)^{P^{\prime \prime}} \psi_{K+1}(K+1) \cdots \psi_{N}(N)\right\} .
\end{aligned}
$$

Therefore, one has the following results: The nodal surfaces of different spin island differ only by different numbering of the coordinates, i.e., go into one another by a suitable number of two-dimensional rotations by $90^{\circ}$.

In one spin island, the nodal surfaces of the $\alpha$ spin states and the $\beta$ spin states are calculated independently; they are cylinders, the former with a $3 K-1$ dimensional cross section and a $3(N-K)$ dimensional axis, the latter with a $3(N-K)-1$ dimensional cross section and a $3 K$-dimensional axis. The axes of these two groups are orthogonal (in the $3 N$-dimensional space) to each other.

That is as far as the author has been able to proceed with certainty. To get further insight consider, as simplest case, that the $\alpha$-part of a spin island contains just two one electron states, $\psi_{1}$ and $\psi_{2}$. Then the corresponding bracket expression in a particular spin island, which is zero on a nodal surface (more exactly, the equation for the 5-dimensional cross section of the cylinder), is

$$
\psi_{2}(1) / \psi_{1}(1)=\psi_{2}(2) / \psi_{1}(2) .
$$

The node of the atomic wave function is only a 4 -fold manifold $\left(z_{1}=0, z_{2}=0\right)$, not a 5 -fold nodal surface.

If the two dimensional surface in three dimensional space

$$
U=f(x, y, z)=\psi_{2} / \psi_{1}
$$

has no maximum or minimum, (11) has as solution one nodal surface. If (12) has one maximum, there are two nodal surfaces, which cross. For more complicated cases the author has not been able to give a satisfactory discussion. 